\title{
Electrochemical array (ECA) as an integrated multi-electrode DNA sensor
}

\author{
Non-member Hiroyoshi Miyahara (TUM Inc.) \\ Non'member Kenichi Yamashita (Kyushu University) \\ Non'member Makoto Takagi (Kyushu University) \\ Non-member Hiroki Kondo (Kyyushu Institute of Technology) \\ Non-member Shigeori Takenaka (Kyushu University)
}

\begin{abstract}
An electrochemical array, ECA, for DNA analysis was designed and constructed as a microarray for the next generation by integrating DNA sensing multiple electrodes. Probe DNAs were immobilized on 25 electrodes of an ECA chip and allowed to detect the target DNA with ferrocenylnaphthalene dimide (FND) as an indicator. As an application example of this system for single nucleotide polymorphisms (SNPs) analysis, a DNA mismatch on the cancer repression gene p53 was successfully analyzed with $88 \%$ precision.
\end{abstract}

Key Words: Electrochernical array (ECA), DNA, Ferrocexylnaphthalene diimide (FND), Single nucleotide polymorphisms (SNPs), p53

\section{Introduction}

The DNA microarray technology is attracting attention as a valuable means of high-throughput analysis of genes in a sample DNA and it may be one of the most useful tools in the postgenome project. This technique consists of the following procedures. ${ }^{1}$ Many different probe DNAs are immobilized on the surface of a glass plate (preparation of a DNA microarray). A labeled DNA sample is allowed to hybridize on the microarray plate. After washing the plate, the sample DNA bound on the plate is detected by the label's signal. However, the following problems remain to be solved in the current DNA microarray technology: (1) supplementary labeling of sample DNA with a fluorescent reagent is necessary and tedious, (ii) quantitative immobilization of probe DNA on different spots of DNA microarray is difficult, and (iii) the efficiency of hybridization on the DNA microarray is variable among different array spots. The DNA chips of Affymetrix Inc, where probe DNAs are synthesized on the silicon chip coupled with a microlithographic technique, have solved problems (ii) and (iii), but (i) still remains.

Electrochemical DNA sensors based on a combination of a probe DNA immobilized on the gold electrode and an electrochemically active higand specific for double stranded DNA are a promising alternative, as a probe DNA can be homogeneously immobilized on the electrode in a controlled fashion and sample DNA needs not to be labeled at all., ${ }^{2,3}$ In addition, they are sensitive enough, quick to run and inexpensive. We designed and synthesized a threading type intercalator FND, highly specific for double stranded DNA to realize a novel DNA sensing system.4? FND binds to the DNA duplex every other base pairs in such a way that the ferrocene moieties are arranged along the DNA groove, assuming a pseudo polyferrocene array. This architecture gives rise to a catalytic current through the electron-conducting pseudo polyferrocene coating the DNA duplex. When this type of DNA sensors is extended to a multi DNA sensor electrode system, i.e., an electrochemical array, ECA, it will be a promising candidate for a practical DNA sensor. In this paper,<smiles>C=C(NCCCN1CCN(CCCN2C(=O)c3ccc4c5c(ccc(c35)C2=O)C(=O)N(CCCN2CCN(CCCNC(=O)C3CCCC3)CC2)C4=O)CC1)C1CC2C=CC1C21C=CC=C1</smiles> 
we constructed an ECA chip composed of 25 electrodes and its basic characteristics were studied concerning the probe DNA immobilization, hybridization, electrochemical detection in the ECA chip coupled with ligand FND.

\section{Experimental}

2.1 Chemicals Ferrocnecarboxylic acid was purchased from Tokyo Kasei Kogyo Co., Ltd. Ferrocenylnaphthalene diimide (FND) is the same as that used previously.47) 5'Mercaptohexyloligonucleotides, HS-GCTITGAGGTGCGTGTTTGT (HS-273C), HS-AGGCTGCTCCCCCCGTGGCC (HSP72), and HS-AGGCTGCTCCCCGCGTGGCC (HS-R72) and oligonucleotides, ACAAACACGCA- CCTCAAAGC (273C) and GGCCACGGGGGGAGCAGCCT (P72-) were custom synthesized by Hokkaido System Science Co., Ltd. These oligonucleotide sequences represent part of the cancer repression gene p53. ${ }^{83}$ The concentrations of these oligonucleotides were estimated from the molar absorptivities. ${ }^{9)}$

2.2 ECA chip ECA chip consists of 25 electrodes with a diameter of each spot of $1.0 \mathrm{~mm}$ and average distance between spots of $4.5 \mathrm{~mm}$ as shown in Fig. 1. Each electrode is covered with a gold galvanizing of a thickness of $2 \mu \mathrm{m}$ (area: $0.79 \mathrm{~mm}^{2}$ ).

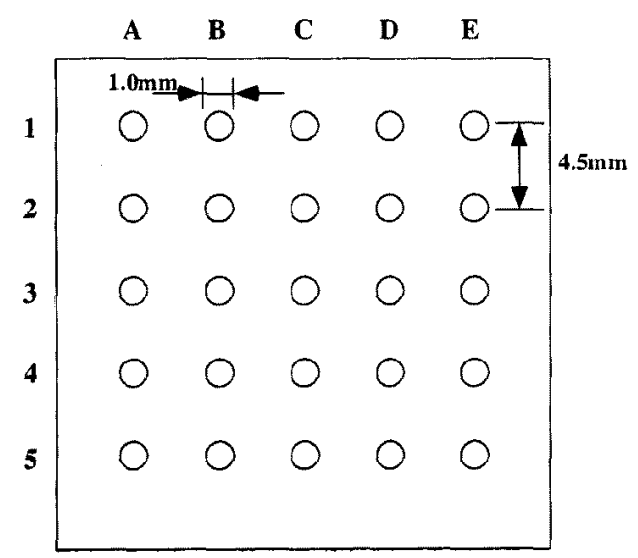

Fig.1. Arrangement and numbering of ECA chip; numerals and alphabets refer to the row and column, respectively.

2.3 Immobilization of probe DNA on the ECA chip Gold electrode parts of ECA chip were soaked in boiling $2 \mathrm{M}$ $\mathrm{KOH}$ for $1 \mathrm{~h}$ and washed with deionized water. These parts were next soaked in concentrated nitric acid, and washed with deionized water and dried by blowing a stream of nitrogen. For chemisorption of a thiolated probe DNA on the gold electrode, one microliter solution of 5'-mercaptohexyloligonucleotide (2.5 pmoll was placed on each gold electrode of an ECA chip and kept in a closed container under high humidity overnight at room temperature.

2.4 Hybridization on an ECA chip After immobilization of probe DNA on the ECA chip, the electrodes were washed with deionized water and allowed to hybridize with 50 pmol of a DNA sample in $2 \times \mathrm{SSC}$ buffer $(33.3 \mathrm{mM}$ sodium citrate buffer containing $33.3 \mathrm{mM} \mathrm{NaCl}$ at $\mathrm{pH} 7.0$ ) for 1 $h$ at room temperature and then washed with the same buffer.

2.5 Electrochemical measurement Electrochemical measurements were performed with ALS Model 610 electrochemical analyzer through the automated multi-converting system (TUM Inc.). Differential pulse voltammetric (DPV) measurement of each electrode of an ECA chip was carried out at room temperature with a normal three electrode configuration consisting of an $\mathrm{Ag} / \mathrm{AgCl}$ reference electrode, a Pt counter electrode, and an indicator electrode on an ECA chip. The data acquisition time in this system was 3.4 $\min (8 \mathrm{~s} \times 25)$. The condition of DPV measurement is the following: initial potential $=0 \mathrm{~V}$, final potential $=0.6 \mathrm{~V}$, scan rate $=100 \mathrm{~m} \mathrm{~V} / \mathrm{s}$, pulse amplitude $=0.05 \mathrm{~V}$, sample width $=16.7 \mathrm{~ms}$, pulse period $=0.2 \mathrm{~s}$, pulse width $=0.05 \mathrm{~s}$, quiet time $=2 \mathrm{~s}$, sensitivity $=10^{-6} \mathrm{AN}$. The electrolytes were $0.2 \mathrm{M}$ phosphate buffer ( $\mathrm{pH} \mathrm{7.0)}$ containing $0.5 \mathrm{mM}$ ferrocenecarboxylic acid to estimate the surface area of the individual electrode of an ECA chip or $0.1 \mathrm{M} \mathrm{AcOH}-\mathrm{AcOK}$ buffer $(\mathrm{pH} 5.6)$ and $0.1 \mathrm{M} \mathrm{KCl}$ carrying $50 \mu \mathrm{M} F \mathrm{FD}$ in the case of DNA analysis.

\section{Results and Discussion}

3.1 Homogeneity of the surface area on the electrode of an ECA chip The DPV of the electrode on the ECA chip was measured in the electrolyte containing $0.5 \mathrm{mM}$ ferrocenecarboxylic acid to evaluate the homogeneity of the surface area on individual electrodes of the ECA chip. Fig. $2 A$ shows an example of the DPV response for electrode E5. The current peak was observed at $300 \mathrm{mV}$ and the shape of the peak and the background current were adequate for DPV measurements. The peak current $\left(i_{\mathrm{pa}}\right)$ for the various electrodes of an ECA chip is shown in Fig. 2B. Statistical treatment of the data gave a mean current of $1064.1 \mathrm{nA}$ with a standard deviation of $73.6 \mathrm{nA}$ or a fluctuation coefficient of $6.9 \%$, showing that the electrodes on the ECA chip are homogeneous enough to be feasible for electrochemical DNA sensing.

3.2 Homogeneity of the amount of the probe DNA immobilized on the individual electrode Thiolated oligonucleotide HS-273C (2.5 pmol) was placed on individual electrodes of an ECA chip to construct a probe DNA-immobilized ECA chip. DPV of the chip was measured in the electrolyte containing $0.05 \mathrm{mM}$ FND. The current peak around $420-460$ $\mathrm{mV}$ was observed for all of the electrodes (Fig. 3A), suggesting concentration of FND to the oligonucleotide region on each electrode. Although FND was developed as an electrochemical indicator of double stranded DNA, the current of FND was observed even with the single stranded DNA-immobilized 
(A)

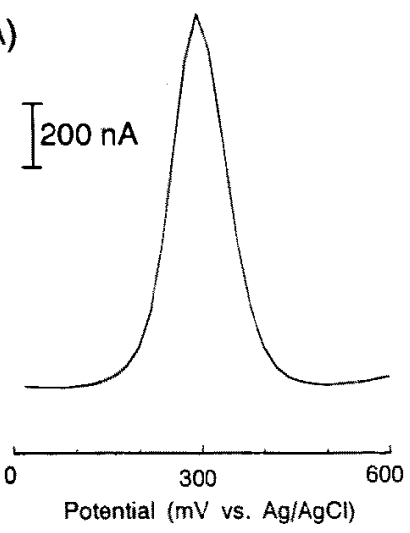

(B)

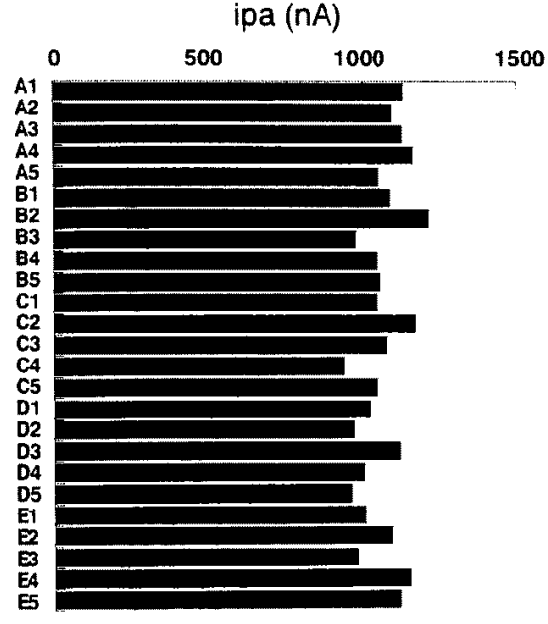

Fig.2. DPV responses of individual electrodes of an ECA chip in the electrolyte containing $0.5 \mathrm{mM}$ ferrocenecarboxylic acid. (A) Example of a DPV curve for electrode E5. (B) The ipa values for all of the electrodes on the ECA chip with a mean of $1064.1 \mathrm{nA}$ and a standard deviation of $73.6 \mathrm{nA}$ or a fluctuation coefficient of $6.9 \%$.

electrodes. This is because an electrostatic interaction occurs between cationic FND and polyanionic single stranded DNA and this property is utilized to evaluate the amount of the single stranded DNA immobilized on the individual electrodes of an ECA chip.

The current io in the single stranded DNA-immobilized electrode increased in proportion to the scan rate, suggesting that it arose from FND bound on the electrode, whereas the io in the presence of ferroœnecarboxylic acid was proportional to the root of the scan rate, suggesting that the diffusion of the ligand limits the current. In addition, as the amount of the thiolated oligonucleotide to be immobilized is reduced, the io value decreased accordingly. As the oligonucleotide length is increased from 20 up to 50 , the io value increased. The io value was independent of the oligonucleotide sequence and the base

電学論 $E ， 121$ 巻 4 号, 平成 13 年 composition as long as the oligonucleotide length is kept constant. These results prove that the io value is a useful measure to evaluate the amount of the single stranded DNA immobilized on the individual electrodes of an ECA chip.

The shape of the DPV peak and stable background of the ECA chip are adequate for DNA sensing. The mean peak current for all the electrodes of the ECA chip was $1114.0 \mathrm{nA}$ with a standard deviation of $208.0 \mathrm{nA}$ (Fig. 3B). A larger error observed for the probe DNA-immobilized electrodes is explained by the fact that the efficiency of DNA immobilization varies considerably from lot to lot.

(A)

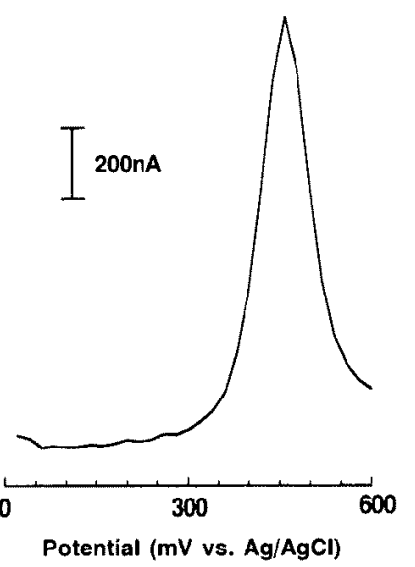

(B)

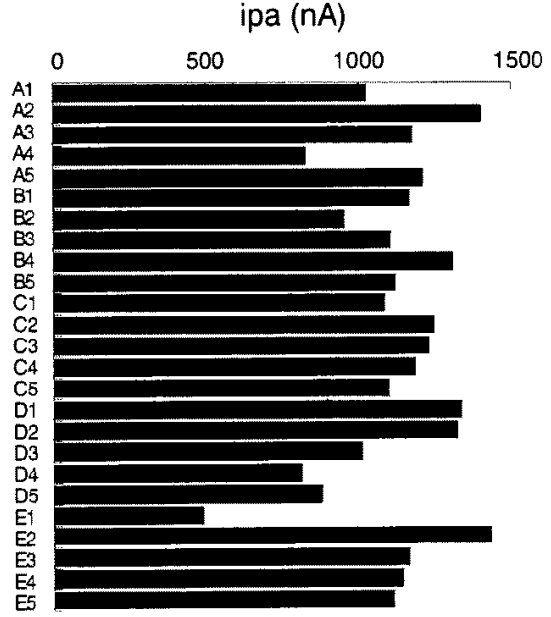

Fig.3. DPV responses of single stranded DNAimmobilized electrodes of an ECA chip. (A) Example of a DPV curve on electrode A1. (B) $I_{p a}$ values for all the electrodes with a mean current of $1114.0 \mathrm{nA}$ and a standard deviation of $208.0 \mathrm{nA}$.

3.3 Current increase by duplex formation: evaluation by $\Delta \mathrm{i}$ An oligonucleotide $273 \mathrm{C}$ immobilized ECA chip was prepared analogously and then DPV was determined before and after hybridization with $50 \mathrm{pmol}$ of the complementary 
oligonucleotide $273 \mathrm{C}$ - in the electrolyte containing $50 \mu \mathrm{M}$ FND. Fig. 4A shows an example of the DPV peak of one electrode on an ECA chip before and after hybridization. The current response corresponding to FND increased after hybridization with $273 \mathrm{C}^{-}$, proving that FND is concentrated on the surface of double stranded DNA more than that of single stranded DNA. This phenomenon can be used for the monitoring of duplex formation on the ECA chip, but the currents for the individual electrodes fluctuated as much as $\mathbf{4 0} \%$ on the ECA chip (see above). Therefore, the $\Delta \mathrm{i}$ value was introduced to correct for this variability. The $\Delta i$ value, defined as $\left(\mathrm{i} / \mathrm{i} 0^{-1}\right) \times 100 \%$, where io and $\mathrm{i}$ refer to the current before and after hybridization, respectively, represents a net increase in the current of the DNA duplex formed per single stranded DNA-immobilized electrode on the ECA chip. Fig. 4B depicts the $\triangle i$ values for all of the electrodes on the ECA chip, giving a mean $\Delta \mathrm{i}$ of $87.3 \%$ with a standard deviation of $29.6 \%$

(A)

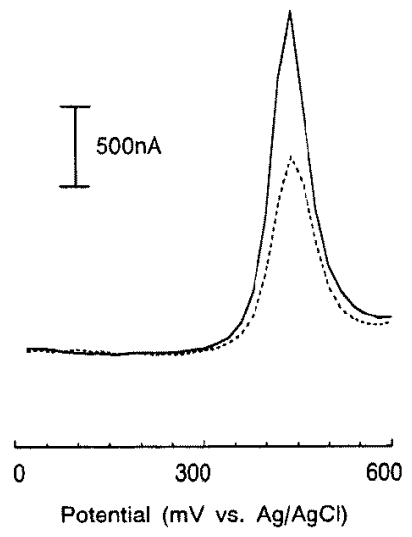

$\Delta i(\%)$

(B)

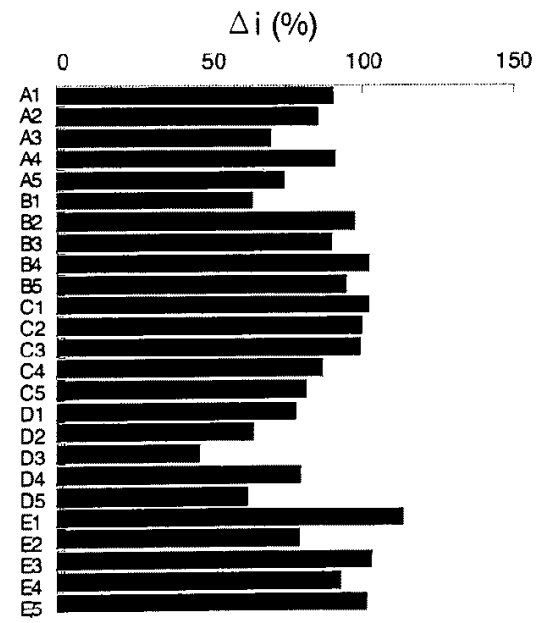

Fig.4. (A) DPV curves for $273 \mathrm{C}$-carrying electrode D1 on an ECA chip before (broken) and after (solid) hybridization with complementary oligonucleotide $273 \mathrm{C}$. (B) $\Delta \mathrm{i}$ values for all the electrodes with a mean of $87.3 \%$ and a standard deviation of $29.6 \%$.
Incidentally, the non-complementary oligonucleotide gave rise to virtually no increase in the currents, demonstrating that the ECA chip is free from non-specific absorption of nucleotides on the electrode.

(A)

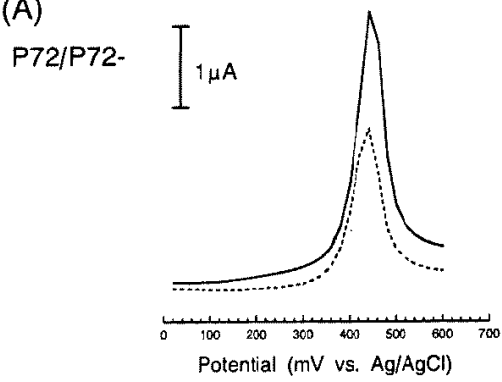

(B)

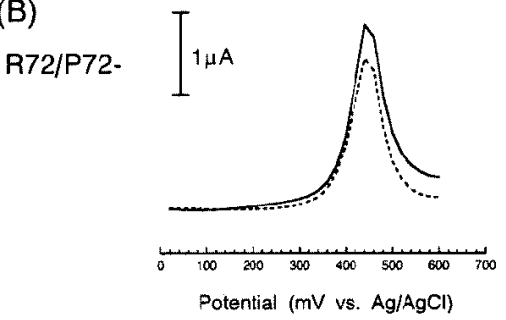

(C)

$\Delta i(\%)$

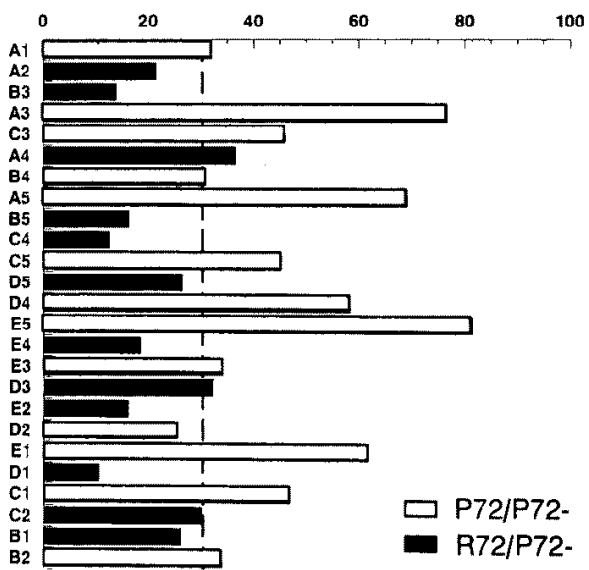

Fig.5. Mismatch detection for the p53 gene. DPV curves for the matched $(\mathrm{P} 72 / \mathrm{P} 72-\mathrm{A})$ and mismatched (R72/P72B) combinations before (broken) and after (solid) hybridization. (C) $\Delta i$ values for the matched (P72/P72) and mismatched (R72/P72.) combinations with a respective mean and standard deviation of $49.3 \%, 18.4 \%$, $21.7 \%$ and $8.4 \%$.

3.4 Mismatch detection of p53 gene Oligonucleotides P72 and R72 were immobilized on the electrode of an ECA chip alternately. They were allowed to hybridize with complementary P72- to form fully matched (P72/P72-, group A) 
complementary P72- to form fully matched (P72/P72-, group A) or G-G mismatched (R72P72-, group B) duplexes. Following washing, DPV of the ECA chip was measured in an electrolyte containing FND at $36^{\circ} \mathrm{C}$. This temperature was chosen after testing various temperatures, i.e., $28,30,32,36$ and $40{ }^{\circ} \mathrm{C}$. It turned out that the $\Delta \mathrm{i}$ value becomes larger at lower temperatures, but the difference in the $\Delta \mathrm{i}$ value between P72/P72- and R72/P72- becomes smaller. The magnitude of $\Delta \mathrm{i}$ values and the difference in $\Delta \mathrm{i}$ value between P72/P72- and R72/P72- become smaller at higher temperatures, thus making $36^{\circ} \mathrm{C}$ the optimal temperature to give the largest difference in $\Delta \mathrm{i}$ between them. Fig. 5A and B depict DPV curves before and after hybridization with P72- in each example of the P72 and R72 immobilized electrodes, respectively. The current increased markedly in the case of the fully matched combination of P72/P72- with a mean $\Delta i$ of $49.3 \%$ and a standard deviation of $18.4 \%$, while the corresponding values for the mismatched were $21.7 \%$ and $8.4 \%$ (Figure $5 \mathrm{C}$ ). These data were independent of the order of measurement. If one assumes rather arbitrarily that the $\Delta \mathrm{i}$ values greater than $30 \%(=49.3 \cdot 18.4,21.7+8.4)$ represent matched, 22 of the 25 electrodes tested or $88 \%$ gave the correct answer. To further assess the reliability of the data, statistical analysis was carried out. First of all, an F test revealed that the data were amenable to Student's $t$ test analysis. The result of such an analysis confirmed that $p$ is smaller than 0.0001 , demonstrating that the difference between the two groups is really significant.

Acknowledgments We thank Ms. N. Katakura and M. Yokoyama for expert technical assistance, and Mr. H. Horiuchi for preparing a glass cell for the ECA chip. This work was supported in part by a grant from the New Energy and Industrial Technology Development Organization (NEDO) of Japan.

(Manuscript received September 5, 2000, revised Nov. 17, 2000)

\section{References}

1) M. Schena ed., DNA Microarrays, Oxford University Press, New York (1999).

2) S. R. Mikkelsen, Electroanalysis, 8, 15 (1996).

3) J. Wang, Anal. Chem., 71, 328R-332R (1999).

4) S. Takenaka, Y. Uto, H. Saita, M. Yokoyama, H. Kondo, and W. D. Wilson, J. Chem. Soc., Chem. Commun., 1111 (1998).

5) S. Takenaka, K. Yamashita, M. Takagi, Y. Uto, and H. Kondo, Denki Kagaku, 66, 1329 (1998).

6) S. Takenaka, K. Yamashita, M. Takagi, Y. Uto, and H. Kondo, Anal. Chem., 72, 1334 (2000).

7) K. Yamashita, M. Takagi, H. Kondo, and S. Takenaka, Chem. Lett., 1038 (2000).
8) C. H. Arrowsmith and P. Morin, Oncogene, 12, 1379 (1996).

9) C. R. Cantor and M. M. Warshaw, Biopolymers, 9, 1059 (1970).

Hiroyoshi Miyahara (Non-member) graduated from the master

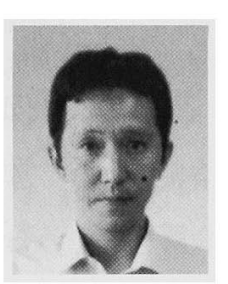
course of Nagoya University in 1989. He is working at TUM Inc. as a research manager. His current research concerns developing ECA chips for gene analysis.

Kenichi Yamashita (Non-member) is a doctoral candidate at

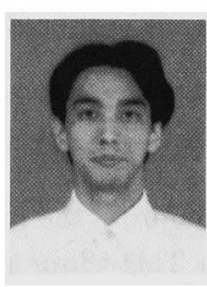
Kyushu University. $\mathrm{He}$ is developing a new DNA sensing technology. Chemical Society of Japan, the Japan Society for Analytical Chemistry, the Molecular Biology Society of Japan member.

Makoto Takagi (Non-member) received his Ph. D. degree in

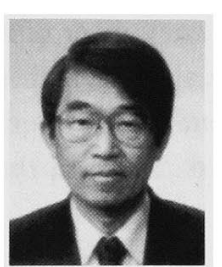
chemistry from Kyushu University in 1966, and is presently professor there. His current research interest is in analytical chemistry with emphasis on molecular recognition. Chemical Society of Japan, the Japan Society for Analytical Chemistry member.

Hiroki Kondo (Non-member) received his Ph. D. degree in

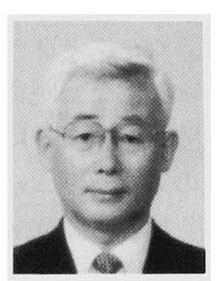
chemistry from Kyushu University in 1972, and is presently professor at Kyushu Institute of Technology. His current research interest is in biologically active substances such as proteins and DNA. Chemical Society of Japan, American Chemical Society member.

Shigeori Takenaka (Non-member) received his $\mathrm{Ph}$. D. degree in

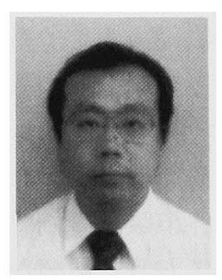
chemistry from Kyushu University in 1988, and is presently associate professor there. His scientific interests are in DNA intercalator chemistry and its medical application. Chemical Society of Japan, the Japan Society for Analytical Chemistry, the Molecular Biology Society of Japan, American Chemical Society member. 